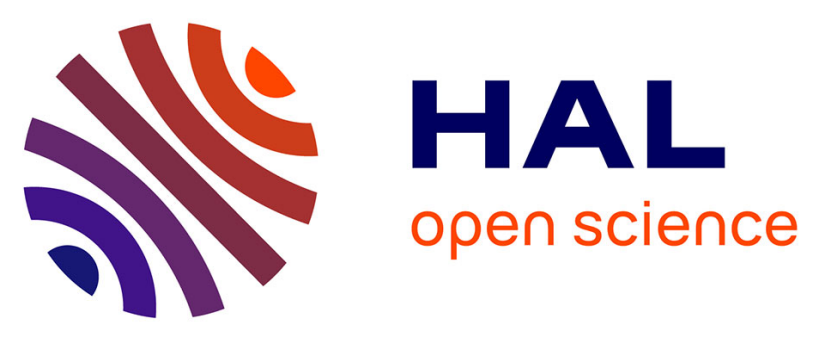

\title{
AMBITION, the Comet Nucleus Cryogenic Sample Return mission for ESA Voyage 2050 program
}

Gianrico Filacchione, Dominique Bockelée-Morvan, Kathrin Altwegg, Eleonora Bianchi, Martin Bizzarro, Jürgen Blum, Lydie Bonal, Fabrizio Capaccioni, Claudio Codella, Mathieu Choukroun, et al.

\section{To cite this version:}

Gianrico Filacchione, Dominique Bockelée-Morvan, Kathrin Altwegg, Eleonora Bianchi, Martin Bizzarro, et al.. AMBITION, the Comet Nucleus Cryogenic Sample Return mission for ESA Voyage 2050 program. Europlanet Science Congress EPSC 2020, Sep 2020, (Virtual Meeting ), Germany. pp.EPSC2020-25. insu-02952276

\section{HAL Id: insu-02952276 \\ https://hal-insu.archives-ouvertes.fr/insu-02952276}

Submitted on 29 Sep 2020

HAL is a multi-disciplinary open access archive for the deposit and dissemination of scientific research documents, whether they are published or not. The documents may come from teaching and research institutions in France or abroad, or from public or private research centers.
L'archive ouverte pluridisciplinaire HAL, est destinée au dépôt et à la diffusion de documents scientifiques de niveau recherche, publiés ou non, émanant des établissements d'enseignement et de recherche français ou étrangers, des laboratoires publics ou privés.

\section{(ㄷ)(i)}

Distributed under a Creative Commons Attribution| 4.0 International License 


\title{
AMBITION, the Comet Nucleus Cryogenic Sample Return mission for ESA Voyage 2050 program
}

\author{
Gianrico Filacchione ${ }^{1}$, Dominique Bockelée-Morvan ${ }^{2}$, and the AMBITION Team* \\ ${ }^{1}$ INAF-IAPS Istituto di Astrofisica e Planetologia Spaziali, via del Fosso del Cavaliere, 100, 00133, Rome, Italy \\ ${ }^{2}$ LESIA, Observatory of Paris, PSL University, CNRS, Sorbonne University, University of Paris, 5 place Jules Janssen, 92195, \\ Meudon Cedex, France \\ ${ }^{*}$ A full list of authors appears at the end of the abstract
}

Since the 1980s, ESA has played a leading role in cometary space science with Giotto and the Rosetta/Philae missions to comets 1P/Halley and 67P/Churyumov-Gerasimenko, respectively. These missions have greatly improved our knowledge of comets and placed Europe in an ideal position for the preparation of future cometary missions. After a cometary flyby mission (Giotto) and an orbiter (Rosetta) with a nucleus lander (Philae), and the selection of F-class Comet Interceptor ESA mission, the next major step in cometary science would be a cryogenic sample return mission as studied in the AMBITION proposal [1] which was submitted to ESA in the context of the future Voyage 2050 program. Despite the success of previous missions, a number of high priority scientific questions are still debated within the cometary community:

- How did cometary materials assemble? What are the building blocks of cometary nuclei?

- Which post-planetesimal evolutionary paths need to be considered? Are comets collisional fragments or primordial planetesimals?

- Which cometary components originate from the time before the formation of the Solar System? What is the nature of the refractory organic materials? How and where did these components form?

- Are there differences in physical/chemical properties in the comet populations? What are the commonalities and differences between comets and primitive asteroids? Are these distinct populations? Do comets, Main Belt comets and active asteroids have different primordial reservoirs?

- How does comet activity work? How do surface and coma observations reconnect with the pristine, deep interior?

- How are the dusty coma, the surrounding plasma, and the nucleus interacting together? Do interactions with the solar wind influence the activity and evolution of comets?

- What contributions did comets make to reservoirs of volatiles and prebiotic compounds on early Earth?

The best approach to answer these questions is to return a pristine and volatile rich cometary sample to Earth. Such a sample, preserved at cryogenic temperature, will be transferred to a stateof-the-art curation facility, where it will be investigated in great detail.

In this scenario part of the sample will be studied by means of different analytical techniques in laboratory-controlled conditions while the remaining sample will be stored at cryogenic temperature 
for future investigations. Laboratory analyses of the returned sample can achieve much higher precision and resolution measurements as compared to in-situ measurements.

The AMBITION study has considered different mission targets (see Figure 1), including Centaurs, Jupiter family comets and its extinct members, returning Oort cloud comets, dynamically new comets, main belt comets and interstellar comets as possible candidates. Among them, Jupiter family comets are the ones which offer the best characteristics, in terms of orbital parameters, evolutionary history, composition and activity, to be to be selected for an L-class cryogenic sample return mission. The other targets could be explored by more consolidated (orbiters, landers) small and medium class missions.

The selection, collection and storage of the sample at controlled cryogenic temperature and pressure during the return flight, Earth re-entry and landing phases will require significant technological advancement to guarantee maintenance of the optimal environmental conditions for the collected sample. Several options are identified for pristine sample collection from the cometary nucleus, including subsurface corers able to extract carrots up to $3 \mathrm{~m}$ depth as well as grabbers and manipulator arms to collect ice-rich, semi-buried boulders from the surface.

The AMBITION mission is the "Holy Grail" in the field of cometary exploration and Solar System formation. For its implementation it will need a strong commitment from the scientific community, industrial partners, ESA and national space agencies.

\begin{tabular}{|c|c|c|c|c|c|}
\hline Comet type & Fly-by & Rendezvous & Landing & Sample return & Cryogenic SR \\
\hline Centaur & M & $M / L$ & $\mathrm{~L}+$ & & \\
\hline Jupiter Family & $\begin{array}{c}\text { DI, DS1, EPOXI } \\
\text { etc. }\end{array}$ & Rosetta & Philae & Stardust & L++ \\
\hline Extinct JFC & $\mathrm{F} / \mathrm{M}$ & $\mathrm{M} / \mathrm{L}$ & L & L & \\
\hline Returning OCC & Giotto etc. & L+ & $\mathrm{L}++$ & & \\
\hline $\begin{array}{l}\text { Dynamically } \\
\text { new }\end{array}$ & $\begin{array}{c}\text { Comet } \\
\text { Interceptor }\end{array}$ & & & & \\
\hline $\begin{array}{l}\text { Main Belt } \\
\text { Comet }\end{array}$ & $M$ & ZhengHe-A & $\mathbf{L}$ & $\begin{array}{c}\text { M (Stardust- } \\
\text { like) } \\
\text { L (surface) }\end{array}$ & \\
\hline $\begin{array}{l}\text { Interstellar } \\
\text { comet }\end{array}$ & $M$ & & & & \\
\hline
\end{tabular}

Figure 1: Approximate mission classes for different mission and comet types, in increasing complexity from left to right, and covering varying evolution stages of comets from the four possible reservoirs (Kuiper Belt, the Oort cloud, the Main Belt, and other planetary systems). Shading indicates approximate cost from yellow (F-class) through orange (M-Class) to red (L-class or multiagency flagship missions). Hatched boxes indicate that such a combination is unfeasible, mostly due to excessive $\Delta v$ requirements. Past and planned missions are shown. From [1].

[1] Bockelée-Morvan, D. et al., AMBITION, Comet Nucleus Cryogenic Sample Return (2019), eprint, 2019arXiv190711081B 


\section{AMBITION Team:}

Kathrin Altwegg, Eleonora Bianchi, Martin Bizzarro, Ju $\square$ rgen Blum, Lydie Bonal, Fabrizio Capaccioni, Claudio Codella, Mathieu Choukroun, Herv $\square$ e Cottin, Bj $\square$ orn Davidsson, Maria Cristina De Sanctis, Maria Drozdovskaya, C $\square$ ecile Engrand, Marina Galand, Carsten G $\square$ uttler, Pierre Henri, Alain Herique, Stavro Ivanovski, Rosita Kokotanekova, Anny-Chantal Levasseur-Regourd, Kelly E. Miller, Alessandra Rotundi, Maria Sch $\square$ onb $\square$ achler, Colin Snodgrass, Nicolas Thomas, Cecilia Tubiana, Stefan Ulamec, Jean-Baptiste Vincent

AMBITION Team: Kathrin Altwegg, Eleonora Bianchi, Martin Bizzarro, Ju $\square$ rgen Blum, Lydie Bonal, Fabrizio Capaccioni, Claudio Codella, Mathieu Choukroun, Herv $\square$ e Cottin, Bj $\square$ orn Davidsson, Maria Cristina De Sanctis, Maria Drozdovskaya, C $\square$ ecile Engrand, Marina Galand, Carsten G $\square$ uttler, Pierre Henri, Alain Herique, Stavro Ivanovski, Rosita Kokotanekova, Anny-Chantal Levasseur-Regourd, Kelly E. Miller, Alessandra Rotundi, Maria Sch $\square$ onb $\square$ achler, Colin Snodgrass, Nicolas Thomas, Cecilia Tubiana, Stefan Ulamec, Jean-Baptiste Vincent 